\title{
Does COPD severity stage change comorbidities pattern?
}

\author{
Sanja Popović-Grle1, Višnja Dukić ${ }^{2 *}$,Josipa Škugor ${ }^{3}$, Dominik Oroz ${ }^{3}$ and Doris Ogresta ${ }^{4}$ \\ ${ }^{1}$ University Hospital Center Zagreb, Clinical Department for Lung Diseases, Jordanovac, Zagreb, Croatia \\ ${ }^{2}$ Special Hospital for Medical Rehabilitation Thalassotherapia Crikvenica, Crikvenica, Croatia \\ ${ }^{3}$ Students, School of Medicine, University of Zagreb, Zagreb, Croatia \\ ${ }^{4}$ Sestre Milosrdnice University Hospital Center, Clinic for Internal Medicine, Zagreb, Croatia
}

\begin{abstract}
Aim: Estimation of the difference in frequency and type of comorbidities in patients with chronic obstructive pulmonary disease (COPD), grouped by COPD severity stages according to the Global Initiative for Chronic Obstructive Lung Disease (GOLD), from 1 to 4.

The data subjects and methods: This study included 168 COPD patients with an equal share of patients in each GOLD stage (GOLD I 47 subjects, GOLD II 43 , GOLD III 41 and GOLD IV 37 subjects). All data subjects, 106 men and 62 women (median age 66), were processed according to the successive arrival at the Clinical Center for Pulmonary Diseases Jordanovac, University Hospital Center Zagreb, for their medical examination.

Results: The most common comorbidities were cardiovascular diseases with $60.1 \%$, regardless of the GOLD stage. These are followed by pneumonia in $25.6 \%$ of COPD patients, anxiety and depression in $16.1 \%$, diabetes in $12.5 \%$ and osteoporosis in $11.9 \%$ of the COPD patients. The most frequent malignant disease was the urinary bladder cancer. The share of the patients who suffered from pneumonia is consistently increasing with the severity of GOLD stages.

Conclusion: The average number of comorbidities in GOLD I stage was 1.5, in GOLD II stage 1.8, GOLD III stage 2.1 and in GOLD IV stage 1.7. This suggests that the difference in the number of comorbidities according to the GOLD stages could be significant. This preliminary result was confirmed by a statistical chi-squared test with a significance level of $5 \%(\alpha=0.05)$, whereas the difference in the types of comorbidities according to the GOLD stages was not statistically significant.
\end{abstract}

\section{Introduction}

Chronic obstructive pulmonary disease (COPD) is a common disease that can be prevented and treated. It is characterized by persistent respiratory symptoms and airflow limitation as a consequence of airway and alveolar abnormalities due to significant exposure to harmful substances or gases. COPD, surprisingly, has already become the third most common cause of death in the world, although, according to the trends, it was expected for it to emerge in third place in 2030 $[1,2]$. The most significant and most studied risk factor for COPD development is tobacco smoking, however the disease can also occur in non-smokers exposed to harmful particles that are inhaled. It is important to emphasize the growing economic and social burden of COPD. The prevalence of COPD in the world is estimated at $11.7 \%$, with approximately three million deaths per year [3].

In the pathogenesis of COPD, the most important mechanisms are chronic inflammation, oxidative stress and imbalance of proteases and antiproteases. The inflammatory process is predominantly present in the airways, lung parenchyma, but it manifests systematically as well. This effect in COPD affects the functioning of other organs (heart, blood vessels, muscles, kidneys, liver, digestive system and brain) [2].

Extrapulmonary comorbidities have also been affecting the prognosis of COPD patients. A common risk factor for numerous comorbidities such as, e.g., coronary heart disease, heart failure, lung cancer and urinary tract cancer (kidney and urinary bladder) is smoking. On the other hand, some other comorbidities such as malnutrition and pulmonary artery disease are the consequences of COPD. The third group of diseases is those with unclear pathophysiological correlation, such as venous thromboembolisms, anxiety, depression, osteoporosis, sleep apnea, anemia and so on. A chronic systemic inflammation is common for most of the extrapulmonary manifestations of COPD [4].

\section{Data subjects and methods}

This is a retrospective study that processed 168 patients with the COPD diagnosis at different stages of the disease according to the GOLD classification, as explained below. The patients were processed according to the chronological order of arrival at the Clinical Department for Lung Diseases Jordanovac, University Hospital Centre Zagreb for their medical examination, from March 2014 to May 2016. Consent of the Ethics Committee of the University Hospital Center Zagreb to conduct the study at the Clinical Center for Pulmonary Diseases Jordanovac "The Study of Chronic Obstructive Pulmonary Disease (COPD) Markers in Induced Sputum, Blood and Urine of Patients with COPD and in Healthy Persons" was issued on 29/10/2013 (Class: 8.1-13/94-2, No.: $02 / 21-J G$ ) and consent with changes in the plan of the study in question on 01/12/2014 (Class: 8.1.-14/84-4, No.: 02/21/JG). The study was commissioned by Fidelta, a Croatian pharmaceutical company for scientific studies in the field of pharmaceuticals that conducted this study pursuant to the agreement with the international pharmaceutical

${ }^{\star}$ Correspondence to: Višnja Dukić, Thalassotherapia Crikvenica - Special Hospital for Medical Rehabilitation Gajevo šetalište 21, 51260 Crikvenica, Croatia, E-mail: visnja.dukic@gmail.com

Key words: chronic obstructive pulmonary disease, comorbidities, outpatients

Received: October 25, 2019; Accepted: November 12, 2019; Published: November 15, 2019 
company Boehringer Ingelheim Pharma GmbH \& Co. KG. All patients signed the informed consent to participate in the aforementioned study. As induced sputum has limitations due to the lung function and is contraindicated if a patient's FEV1 is below $0.75 \mathrm{~L}$, very severe COPD patients were less represented in the quoted study. For the same reason hospitalized patients with COPD exacerbations at the Clinic for Pulmonary Diseases Jordanovac, University Hospital Center Zagreb, with previously known very severe COPD were included during the same period.

In accordance with the GOLD classification of airflow limitation severity in patients with FEV1/FVC below 0.70, taking into account the post-bronchodilator FEV1 values, our patients were divided into 4 groups: GOLD1 - mild airflow limitation severity, FEV1 is $80 \%$ or higher than the predicted value, GOLD2 - moderate airflow limitation severity, FEV1 is at $80-50 \%$ of the predicted value, GOLD3 - severe airflow limitation severity, FEV1 is $50-30 \%$ of the predicted value, and GOLD 4 - very severe airflow limitation severity, FEV1 is lower than $30 \%$ of the predicted value. Among 168 patients, the share of patients in each GOLD stage was similar (Figure 1). The male gender is more common than the female (Figure 1), and the age of the patients ranges from 50 to 83 , with an average age of 66 .

\section{Statistics}

The chi-squared test was used for the statistical analysis. TEST 1 The number of comorbidities per GOLD stages: Alternative hypothesis $H 1$ was accepted: the number of comorbidities varies by GOLD stages $(p=0.0016<\alpha=0.05$ statistically significant). TEST 2 - Type of comorbidities per GOLD degrees: Null hypothesis $H 0$ was accepted: the type of comorbidities does not vary by GOLD degrees $(p=0.1736$ $>\alpha=0.05$ not statistically significant) (Table 1 ).

\section{Results}

Out of 168 patients, 149 of them (89.1\%), besides COPD, have one comorbidity or more. In the GOLD I group $91.5 \%$ have comorbidities, in the GOLD II group $81.4 \%$, in the GOLD III group $95.1 \%$ and in the GOLD IV group $83.8 \%$. According to the obtained result, the number of comorbidities by GOLD stages increases proportionally to the severity of the disease (Figure 2). Statistical tests confirmed that the difference in the number of comorbidities by GOLD stages is significant $(\mathrm{p}=0.0016)$, while the difference in types of comorbidities by GOLD stages is not statistically significant $(p=0.1736)$. Further, the comorbidities according to frequency are shown, regardless of the GOLD stage (Figure 3), as well as the most frequent comorbidities in each of the GOLD stages (Figure 4).
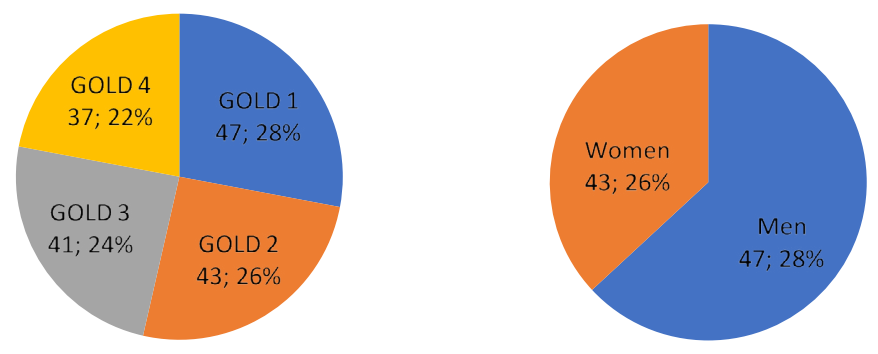

Figure 1. Number and ratio of patients by GOLD groups (left) and by gender (right)

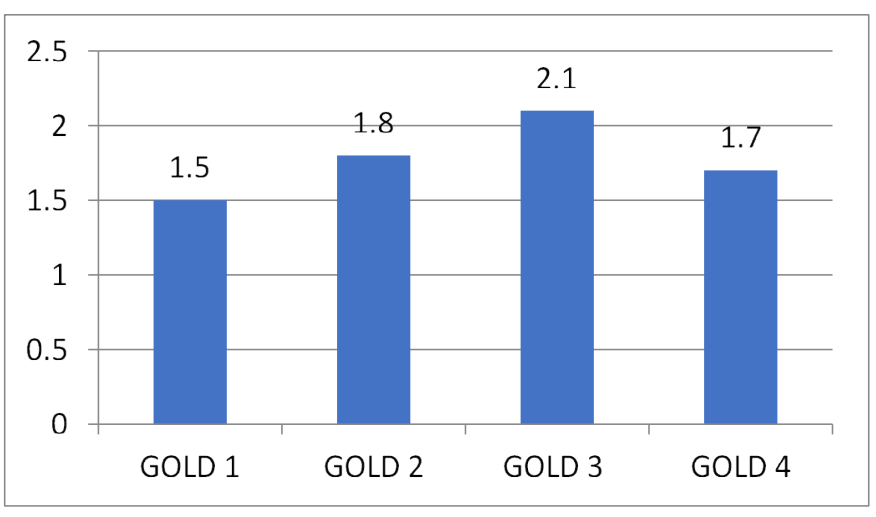

Figure 2. The number of comorbidities by GOLD groups

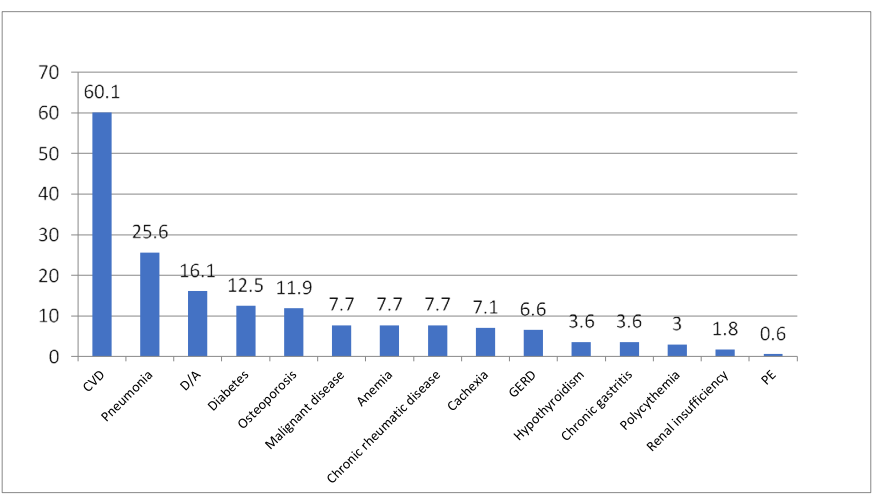

Figure 3. The representation of comorbidities by frequency, regardless of GOLD stage 1. Cardiovascular diseases (CVD); 2. Pneumonia; 3. Depression and anxiety (D/A); 4. Diabetes; 5. Osteoporosis; 6. Malignant disease; 7. Anemia; 8. Chronic rheumatic diseases; 9. Cachexia; 10. Gastroesophageal reflux disease (GERD); 11. Hypothyroidism; 12 Chronic gastritis; 13. Polycythemia; 14. Renal insufficiency; 15. Pulmonary embolism (PE)

Table 1. Chi-square statistics, number of degrees of freedom and $p$ value for both tests

\begin{tabular}{|c|c|c|}
\hline $\begin{array}{c}\text { Chi-squared test } \\
\text { (significance level } \boldsymbol{\alpha}=\mathbf{0 . 0 5})\end{array}$ & TEST 1 & TEST 2 \\
\hline Chi-square statistics $\left(\chi^{2}\right)$ & 31.7 & 50.5 \\
\hline Degrees of freedom $(\mathrm{df})$ & 12 & 42 \\
\hline p value $(\mathrm{P})$ & 0.0016 & 0.1736 \\
\hline
\end{tabular}

\section{Discussion}

In this study $89.1 \%$ of patients had comorbidities. In a systematic review of large clinical studies and observational studies, $79.4 \%$ and 74.2\% patients had comorbidities, respectively [5].

So far, the Charlson Comorbidity Index - CCI has been used in practice and in research for the assessment of the 10-year survival of patients with multiple comorbidities. The patient's age as well as the type, number and the severity of comorbidities are taken into account [6].

Another important index is the COMCOLD (COMorbidities in Chronic Obstructive Lung Disease), an index that focuses on the quality of life in COPD patients. This index consists of depression, anxiety, peripheral arterial disease, cerebrovascular disease and symptomatic heart disease [7].

\section{Cardiovascular comorbidity}

In this study, $60.1 \%$ of patients had been diagnosed with some of the cardiovascular diseases. Most of them, 78\%, had arterial hypertension, 


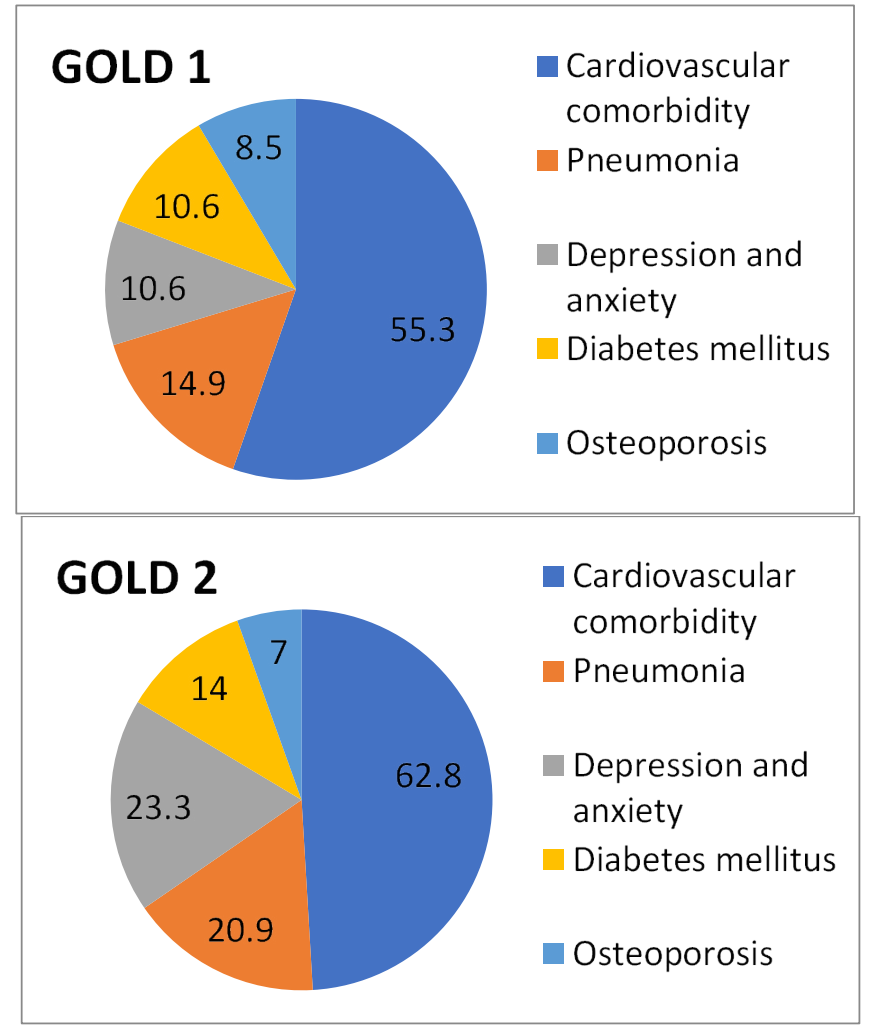

\section{GOLD 3}

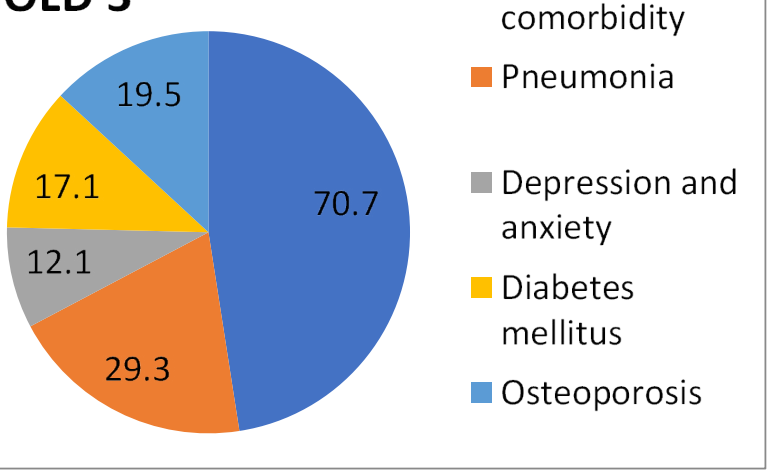

\section{GOLD 4}

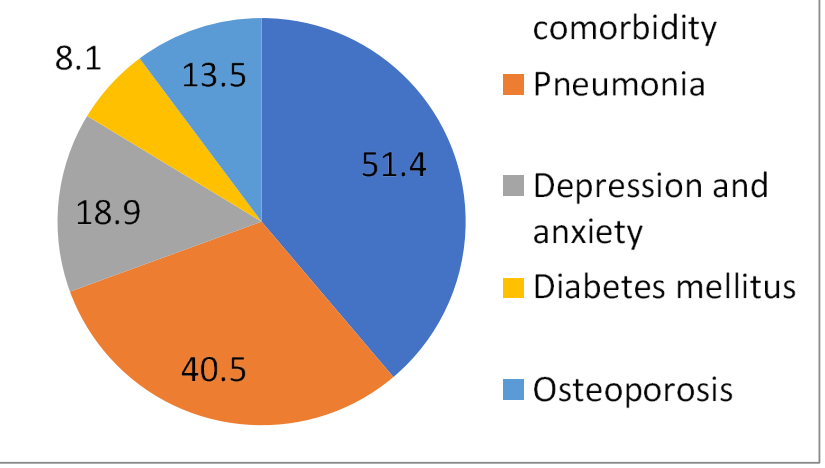

Figure 4. The representation of the most frequent comorbidities in percentages, by GOLD stages
5.9\% the ischemic heart disease and $7.1 \%$ had arrhythmia. The cardiovascular comorbidity was the most frequent among all patient groups, from GOLD I to GOLD IV group.

In the systematic review of large observational studies, the frequency of hypertension is $40-60 \%$, of ischemic heart disease $12.5-41 \%$ and of arrhythmia $11.3-15.8 \%$ [8].

This group of diseases represents the expected most frequent comorbidity in COPD patients, given that the major risk factors are old age, obesity and tobacco smoking. The underlying pathophysiological mechanisms are likely to be triggered by endothelial dysfunction and coagulopathy due to systemic inflammation. The cardiovascular comorbidity has a direct impact on the survival of COPD patients [4].

\section{Pneumonia}

In our study, $25.6 \%$ of patients had pneumonia as a comorbidity, and the number of pneumonia cases consistently increased with the stage of COPD severity according to the GOLD classification.

According to a cohort study conducted on over 40 thousand patients, the incidence of community-acquired pneumonia in COPD patients was $22.4 \%$ and the pneumonia incidence increased with the severity stage of COPD [9]. According to the same study, the risk factors for community-acquired pneumonia are age over 65 , congestive heart failure, prior exacerbations of COPD that required hospitalization and severe COPD on a long-term oxygen therapy or therapy applied via a nebulizer. Pneumonias are much more frequent in COPD patients than in the general population, i.e., these patients have a four times greater risk of developing pneumonia, regardless of their smoking status [10].

\section{Depression and anxiety}

Depression and anxiety are equally frequent in all GOLD stages in the examined group of patients and have been recorded in a total of $16.1 \%$ of our patients. According to earlier studies, depending on the study, COPD stage and the scale used for assessment, depression occurs in $20-60 \%$ of COPD patients and is more frequent in COPD patients than in patients with other chronic diseases [4].

Depression and anxiety occur more often among younger patients, women and patients with predominant symptoms related to the airways. According to one study, patients with the anxiety and/or depression comorbidities will be hospitalized for COPD earlier, and it is possible that these patients experience dyspnea more intensely and earlier than other patients. According to the longitudinal studies, the depression diagnosis doubles 12 months after the COPD diagnosis $[4,11]$. According to the aforementioned COTE and COMCOLD indices for mortality prediction, i.e., for the assessment of quality of life of the COPD patients, anxiety plays a very important role [9].

\section{Diabetes mellitus}

$12.5 \%$ of patients in the examined group have diabetes mellitus, which is in accordance with the previous data from the scientific literature. We did not determine an increase in prevalence with the progression of COPD severity.

In a national study there were $12.7 \%-16.3 \%$ of diabetics among the COPD patients, which is significantly higher than in the general population [12]. According to the same study, the patients in GOLD stages III and IV had a higher diabetes prevalence.

According to the meta-analyses, the association of active and passive smoking with a significantly higher risk of developing type 2 
diabetes was confirmed. Given the importance of impacts of smoking in COPD patients, the prevalence of diabetes among the COPD patients is not surprising $[13,14]$.

\section{Anemia and polycythemia}

In the examined group, $7.7 \%$ of patients had anemia and $3 \%$ of patients had polycythemia. Polycythemia is expected in hypoxemic smokers; however, the ratio of anemia and polycythemia is in accordance with the current knowledge of far greater frequency of anemia compared to polycythemia in COPD patients. According to the literature, the prevalence of anemia in COPD patients ranges from 4.9 to $38 \%$, and, alongside COPD, it represents an independent risk factor of reduced functional capacity, whereas the prevalence of polycythemia is low $(6 \%$, i.e., $8.4 \%)$, and is not associated with a less favorable outcome for the patient. It should be noted that severely ill COPD patients were treated with oxygen therapy [15-17].

In our patients, anemia was not more frequent with a more severe COPD stage, however it is evident that all our patients with anemia were men older than 60 and besides anemia they had other comorbidities, usually two or more. The result is consistent with a large retrospective cohort study on 2404 COPD patients, according to which elderly male patients with multiple comorbidities and non-Caucasian patients were more prone to anemia [18].

Anemia in COPD is considered to be anemia of chronic disease, but the mechanisms of its occurrence have not yet been fully clarified.

\section{Malignant diseases}

In the examined group, $7.7 \%$ of patients had a malignant disease. One patient had lung cancer $(0.6 \%)$, while five of them had urinary bladder cancer ( $3 \%$ of the examined group).

According to one study, lung cancer is four times more common in COPD patients than in the general population, i.e., up to $0.6 \%$ of men and up to $0.5 \%$ of women, compared to the general population where the frequency of lung cancer is estimated at approximately $0.1 \%$ in both genders [19]. According to a cohort study conducted in France, $15 \%$ of COPD patients had lung cancer, and $11 \%$ of men had a urinary bladder cancer, which is the third most frequent cancer after prostate cancer and lung cancer [20]. In another study, 9.1\% of patients had lung cancer, and $3.1 \%$ of COPD patients from the examined group had kidney and urinary bladder cancer.

In an American cohort study, it was found that smoking is a strong risk factor for urinary bladder cancer in both genders. Compared with non-smokers, smokers have a 4.06 times higher risk of urinary bladder cancer, while former smokers have 2.22 times higher risk [21].

\section{Rheumatic diseases}

In our study, $7.7 \%$ of patients had a rheumatic disease. According to a prospective, multi-centric observational study, $28.7 \%$ of the observed COPD patients had a degenerative joint disease [9]. The data on the frequency of this comorbidity is not surprising given that obesity, age, gender, race, genotype, bone density, smoking (so far inconsistent evidence) and muscular strength are listed as the risk factors for osteoarthritis [22].

\section{Cachexia}

Among our patients, $7.1 \%$ had pulmonary cachexia, but the trend of cachexia frequency increase in relation to the degree of severity of the disease was not confirmed.
According to the Danish study, the incidence of malnutrition was more frequent in patients at stages III and IV than at stages I and II of the disease. In a Dutch study, 11\% of COPD outpatients had a low body mass index (BMI), but there was $15 \%$ of patients with a normal BMI, however with a low-fat free mass index (FFMI) [23]. Because of its prognostic importance, $\mathrm{BMI}$ is one of the four elements of the previously mentioned BODE index - beside the degree of airflow obstruction, the degree of dyspnea and the result in a 6-minute walk test.

\section{Gastroesophageal reflux disease (GERD)}

In our group of patients, GERD was recorded in $6.6 \%$ of data subjects.

According to the scientific literature, as self-assessed by patients, the proportion of patients with GERD reaches $29 \%$, and after $\mathrm{pH}$ measurement the number of proven refluxes amounts to as much as $50 \%$ of COPD patients. The sensitivity of COPD patients to reflux disease may be particularly pronounced due to accentuated intrathoracic pressure variations, frequent coughing, diaphragmatic flattening and the use of beta2-agonists. According to the existing literature, GERD alongside COPD is associated with more pronounced symptoms, poorer quality of life related to health and increased exacerbation frequency, and proton-pump inhibitors might prevent acute exacerbations of COPD and might do the gastroprotection in patients frequently using nonsteroidal antirheumatics or corticosteroids [24,25].

\section{Hypothyroidism}

$3.6 \%$ of our patients have hypothyroidism, which is similar to the general public prevalence of 3.05\% [26], however in the Divo et al. study, approximately $7 \%$ of the patients with COPD had hypothyroidism [9]. According to one study on COPD patients with thyroid dysfunction, a significant disorder was observed in the maximal inspiratory pressure and in the maximal expiratory pressure values, furthermore a negative correlation between hypoxemia and the thyroid-stimulating hormone was also noted [27].

\section{Kidney failure}

$1.8 \%$ of our patients had kidney failure, while the studies recorded a frequency of $16.7 \%$ [8], i.e., $10.2 \%$ [28]. According to a systematic review of 9 studies, it was found that COPD patients have 2.2 times higher chances of developing chronic kidney disease (CKD) than patients in the control group [29]. Kidney complications in COPD patients are especially frequent in patients with hypoxemia and hypercarbia [30].

\section{Pulmonary embolism (PE)}

$0.6 \%$ of our patients had pulmonary embolism (PE). In the Divo et al. cohort study [9], $2.1 \%$ of COPD patients had PE. The frequency of venous thromboembolism (VTE) in COPD patients is explained by three factors of the Virchow's triad, i.e., with the presence of systemic venous endothelial dysfunction, coagulopathy and venous stasis due to physical inactivity.

\section{Conclusion}

COPD has a high prevalence of accompanying comorbidities, with cardiovascular diseases in the first place by incidence, as we have confirmed in this research. For the given sample of patients, we have proved that the difference in the number of comorbidities according to the GOLD stages is statistically significant, whereas the difference in the types of comorbidities according to the GOLD stages is not statistically significant. 
When evaluating patients with COPD, the frequency, type and significance of comorbidities should be kept in mind. It is important to recognize and treat comorbidities in a timely manner in order to achieve positive effects on the course of the disease, life expectancy and quality of life of a COPD patient.

\section{References}

1. GBD 2016 Causes of Death Collaborators (2017) Global, regional, and national agesex specific mortality for 264 causes of death, 1980-2016: a systematic analysis for the Global Burden of Disease Study 2016. Lancet 390: 1151-1210. [Crossref]

2. The Global Strategy for the Diagnosis, Management and Prevention of COPD (2018) Global Initiative for Chronic Obstructive Lung Disease (GOLD).

3. Adeloye D, Chua S, Lee C, Basquill C, Papana A, et al. (2015) Global Health Epidemiology Reference Group (GHERG). Global and regional estimates of COPD prevalence: Systematic review and meta-analysis. J Glob Health 5: 020415. [Crossref]

4. Cavaillès A, Brinchault-Rabin G, Dixmier A, Goupil F, Gut-Gobert C, et al. (2013) Comorbidities of COPD. Eur Respir Rev 22: 454-475. [Crossref]

5. Akter S, Goto A, Mizoue T (2017) Smoking and the risk of type 2 diabetes in Japan: A systematic review and meta-analysis. J Epidemiol 27: 553-561. [Crossref]

6. Charlson Comorbidity Index (2018) https://www.mdcalc.com/charlson-comorbidityindex-cci.

7. Frei A, Muggensturm P, Putcha N, Siebeling L, Zoller M, et al. (2014) Five comorbidities reflected the health status in patients with chronic obstructive pulmonary disease: the newly developed COMCOLD index. J Clin Epidemiol 67: 904-911. [Crossref]

8. Miravitlles M, Price D, Rabe KF, Schmidt H, Metzdorf N, et al. (2015) Comorbidities of patients in tiotropium clinical trials: comparison with observational studies of patients with chronic obstructive pulmonary disease. Int J Chron Obstruct Pulmon Dis 10: 549-564. [Crossref]

9. Muellerova H, Chigbo C, Hagan GW (2012) The natural history of communityacquired pneumonia in COPD patients: A population database analysis. Respir Med 106: $1124-1133$

10. Braeken DCW, Rohde GGU, Franssen FME (2017) Risk of community-acquired pneumonia in chronic obstructive pulmonary disease stratified by smoking status: a population-based cohort study in the United Kingdom. Int J Chron Obstruct Pulmon Dis 12: $2425-2432$.

11. Brown JP, Martinez CH (2016) Chronic obstructive pulmonary disease comorbidities. Curr Opin Pulm Med 22:113-118.

12. Mannino DM, Thorn D, Swensen A (2008) Prevalence and outcomes of diabetes, hypertension and cardiovascular disease in COPD. Eur Respir J 32: 962-969.

13. Maddatu J, Anderson-Baucum E, Evans-Molina C (2017) Smoking and the risk of type 2 diabetes. Transl Res 184: 101-107. [Crossref]
14. Cote C, Zilberberg MD, Mody SH, Dordelly LJ, Celli B (2007) Haemoglobin level and its clinical impact in a cohort of patients with COPD. Eur Respir J 29: 923-929. [Crossref]

15. John M, Lange A, Hoernig S (2006) Prevalence of anemia in chronic obstructive pulmonary disease: comparison to other chronic diseases. Int J Cardiol 111: 365-370.

16. Chambellan A, Chailleux E, Similowski T (2005) ANTADIR Observatory Group. Prognostic value of the hematocrit in patients with severe COPD receiving long-term oxygen therapy. Chest 128: 1201-1208.

17. Shorr AF, Doyle J, Stern L (2008) Anemia in chronic obstructive pulmonary disease: epidemiology and economic implications. Curr Med Res Opin 24: 1123-1130.

18. Kiri VA, Soriano J, Visick G, Fabbri L (2010) Recent trends in lung cancer and its association with COPD: an analysis using the UK GP Research Database. Prim Care Respir J 19: 57-61. [Crossref]

19. Laforest L, Roche N, Devouassoux G (2016) Frequency of comorbidities in chronic obstructive pulmonary disease, and impact on all-cause mortality: A population based cohort study. Res Med.

20. Freedman ND, Silverman DT, Hollenbeck AR (2011) Association between smoking and risk of bladder cancer among men and women. JAMA 306: 737-745.

21. Litwic A, Edwards MH, Dennison EM, Cooper C (2013) Epidemiology and burden of osteoarthritis. Br Med Bull 105: 185-199. [Crossref]

22. Vermeeren MA, Creutzberg EC, Schols AM, Postma DS, Pieters WR, et al. (2006) Prevalence of nutritional depletion in a large out-patient population of patients with COPD. Respir Med 100: 1349-1355. [Crossref]

23. Martinez CH, Okajima Y, Murray S (2014) Impact of self-reported Gastroesophageal reflux disease in subjects from COPDGene cohort. Respir Res 15: 62.

24. Vanfleteren LEGW, Spruit MA, Wouters EFM (2016) Management of chronic obstructive pulmonary disease beyond the lungs. Lancet Respir Med.

25. Madariaga AG, Palacios SS, Guillén-Grima F (2014) The Incidence and Prevalence of Thyroid Dysfunction in Europe: A Meta-Analysis. The Journal of Clinical Endocrinology \& Metabolism 99: 923-931.

26. Terzano C, Romani S, Paone G, Conti V, Oriolo F (2014) COPD and thyroid dysfunctions. Lung 192: 103-109. [Crossref]

27. Fedeli U, De Giorgi A, Gennaro N, Ferroni E, Gallerani M, et al. (2017) Lung and kidney: a dangerous liaison? A population-based cohort study in COPD patients in Italy. Int J Chron Obstruct Pulmon Dis 12: 443-450. [Crossref]

28. Gaddam S, Gunukula SK, Lohr JW, Arora P (2016) Prevalence of chronic kidney disease in patients with chronic obstructive pulmonary disease: a systematic review and meta-analysis. BMC Pulm Med 16: 158. [Crossref]

29. Mapel D (2014) Renal and hepatobiliary dysfunction in chronic obstructive pulmonary disease. Curr Opin Pulm Med 20: 186-193. [Crossref]

Copyright: (C2019 Popović-Grle S. This is an open-access article distributed under the terms of the Creative Commons Attribution License, which permits unrestricted use, distribution, and reproduction in any medium, provided the original author and source are credited. 